\title{
Article \\ Fully Metallic Reflectarray for the Ku-Band Based on a 3D Architecture
}

\author{
Jaime Velasco, Ignacio Parellada-Serrano and Carlos Molero *D
}

check for updates

Citation: Velasco, J.; ParelladaSerrano, I.; Molero, C. Fully Metallic Reflectarray for the Ku-Band Based on a 3D Architecture. Electronics 2021, 10, 2648. https://doi.org/10.3390/ electronics10212648

Academic Editor: Giovanni Andrea Casula

Received: 29 September 2021

Accepted: 26 October 2021

Published: 29 October 2021

Publisher's Note: MDPI stays neutral with regard to jurisdictional claims in published maps and institutional affiliations.

Copyright: (c) 2021 by the authors. Licensee MDPI, Basel, Switzerland. This article is an open access article distributed under the terms and conditions of the Creative Commons Attribution (CC BY) license (https:// creativecommons.org/licenses/by/ $4.0 /)$.
Department of Signal Theory, Telematics and Communications, University of Granada, 18071 Granada, Spain; jaimevelgar@correo.ugr.es (J.V.); parellada@ugr.es (I.P.-S.)

* Correspondence: cmoleroj@ugr.es

\begin{abstract}
This document presents the design and manufacture of a reflectarray (RA) antenna for the $\mathrm{Ku}$-band that is based on a fully-metallic 3D architecture. The reflectarray unit cell is formed by a square-shaped waveguide section ending in a short circuit, which is the reflectarray back ground plane. Each cell has the ability of configuring the phase of its own reflected field by means of resonators perforated on the walls of the cell waveguide section. The resonator-based waveguide cell introduces the 3D character to the design. The geometry of the resonators and the size variation introduces the phase behavior of each cell, thus, conforming the radiation pattern of the reflectarray. This design explores the potential of phase value truncation (six states and two states) and demonstrates that proper pattern results can be obtained with this phase truncation.
\end{abstract}

Keywords: reflectarray; 3D architecture; resonator-based waveguide cells; satellite communications; Ku-band

\section{Introduction}

The increasing requirements in antennas for broadcast and telecommunications are becoming challenging due to the emergence of novel technologies and the fast development of new fabrication techniques - in particular, the arrival of the 5G communications standard and the establishment of operating bands at millimeter frequencies [1]. Microwave devices operating at these frequencies find several restrictions associated with size, weight, fabrication constraints or a relevant increase of losses [2].

An essential element in current satellite infrastructures is beamforming networks [3]. Parabolic antennas widely cover this task-in particular, beam-redirection and focusing. However, they have intrinsic drawbacks as very large dimensions, bulkiness and heavy weight [4], and their use is not suitable in several scenarios. The common alternative to parabolic reflectors are phased array antennas [5-8]. Phased array antennas are, in contrast to the parabolic ones, planar devices. They are formed by distributions of individual emitters whose field amplitudes and phases are externally controlled. The global radiation pattern of a phased array antenna is obtained by superposing the contribution of the individual fields radiated from each emitter [9].

Phased arrays exploit the ability to steer a radiation beam into a desired direction. They may include the feeding network in the device architecture, which is generally supported on complex circuitry. For instance, they require the use of power dividers [10], phaseshifters [11] or active elements [12]. Reflectarray (RA) antennas are a less complex solution of phasing systems, being lightweight, low-profile and showing adaptability [4,13]. To the author's knowledge, the first RA design was reported in the sixties by D. Berry et al. [14]. At first, RA prototypes were typically based on metallo-dielectric structures [15-18], fabricated via standard PCB techniques.

However, due to the growth of novel 3D-printing fabrication techniques, RAs based on fully-dielectric [19] and fully-metallic designs [20] have emerged. In particular, fully metallic RAs are being demanded beyond the K- and Ka-bands, where dielectric losses 
stop being negligible. Examples of fully metallic RAs have already been proposed and published in the literature, such as those reported in [21-23].

The fast and recent development of 3D-printing techniques introduces the possibility of innovating in the shape of the cells [24]. For instance, 3D architectures arise as elegant solutions due to the additional degrees of freedom they introduce [25]. The 3D character of a given cell is forced by including resonant elements along the axial (or propagation) direction $[26,27]$. 3D cells have the ability to independently control different field components. Paradigmatic examples were reported in [28,29], where fully-metallic cells with 3D architectures were employed to design dual-band/dual-pol devices, combining both 3D architecture and full-metal cavities. These type of cells are promising since they are mechanically self-consistent, and no help of support is needed.

The use of fully-metallic structures is justified due to the need for the avoidance of dielectric materials. Higher frequencies increase the dielectric-losses importance, which is directly responsible of the performance degradation in gain or insertion losses [30,31]. Space applications do not recommend the employment of dielectric materials owing to the extreme conditions they must support in spacial environments [32,33].

In this work, we present a fully-metallic RA based on the ideas reported in [29]. Though our final goal is the conception of a RA operating at millimeter frequencies, in this document, we focus on a Ku-band prototype with the aim of obtaining a first proof of concept. The RA is supported on a metallic metasurface consists of distributions of square waveguides. The structure is illuminated by a normally-oriented plane wave. The waveguide walls are perforated with resonators, whose role is to modify/control the individual phase of the reflected wave associated with each of the waveguide cells.

Under these circumstances, two kinds of RAs are presented. First, a RA formed by a discrete distribution combining six possible phase states; and a second case considering binary cells, highly used currently due to their easy implementation [34]. The aim of the present paper is to show the functioning of the structure in the passive configuration. A future goal is to attain reconfigurability as was proposed in [35]. Reconfigurability is conceived to be via graphene oxide, which is a promising switcher.

This document is organized as follows: Section 2 describes the principle of operation in terms of the unit cell. Section 3 shows the performance of the cell for different geometries. Section 4 includes a numerical validation of the concept, for both prototypes with two and six possible states. In Section 5, we explain the fabrication process and the experimental results obtained in the laboratory. Finally, our conclusions for this work are drawn in Section 6.

\section{Brief Description of the Unit-Cell Type}

The structure under consideration in the present manuscript is a Frequency Selective Surface (FSS) based on a full-metal design. As shown in Figure 1a, it consists of a twodimensional arrangement of classical square waveguides (cells). This type of architecture was previously reported in $[29,36]$, functioning as polarizers and pass-band prototypes. The dimensions of the cells are such that no propagating modes are excited at the operation frequency ( $P \leq \lambda_{0} / 2$ with $\lambda_{0}$ being the wavelength in vacuum). Instead, only evanescent modes will be present inside the cell. A ground plane is placed at the bottom of the cell, in order to force full-reflection response.

Perforations with special shape are done on the waveguide walls with the purpose of controlling the field behavior inside the cell. These perforations actually play the role of slot resonators, which drastically modify the electromagnetic response of the cell at frequencies close to their own resonance frequency. Two main resonator-shapes are proposed, illustrated in Figure 1b,c. Figure 1b shows a C-shaped perforation. Similarly, a S-shaped resonator was perforated on the wall of the cell in Figure 1c. Their effective length $L$ is assumed to be long in comparison with the waveguide dimensions.

The resonators are extended along the propagation (axial) direction $z$, giving a 3D character to the structure. 3D architectures enhance certain typical properties of 2D FSSs 
designs. The inclusion of resonant elements along the propagation direction introduces an additional degree of freedom, which is useful for design and optimization processes. Moreover, the control of the vertical and horizontal field-polarizations (V- and H-pol, respectively) can be rigorously decoupled. In this sense, the resonators on the walls inside the YZ-plane manipulate V-pol and are completely opaque for H-pol. The structure here proposed is optimal for V-pol incidence. More information about this issue is reported in [29].

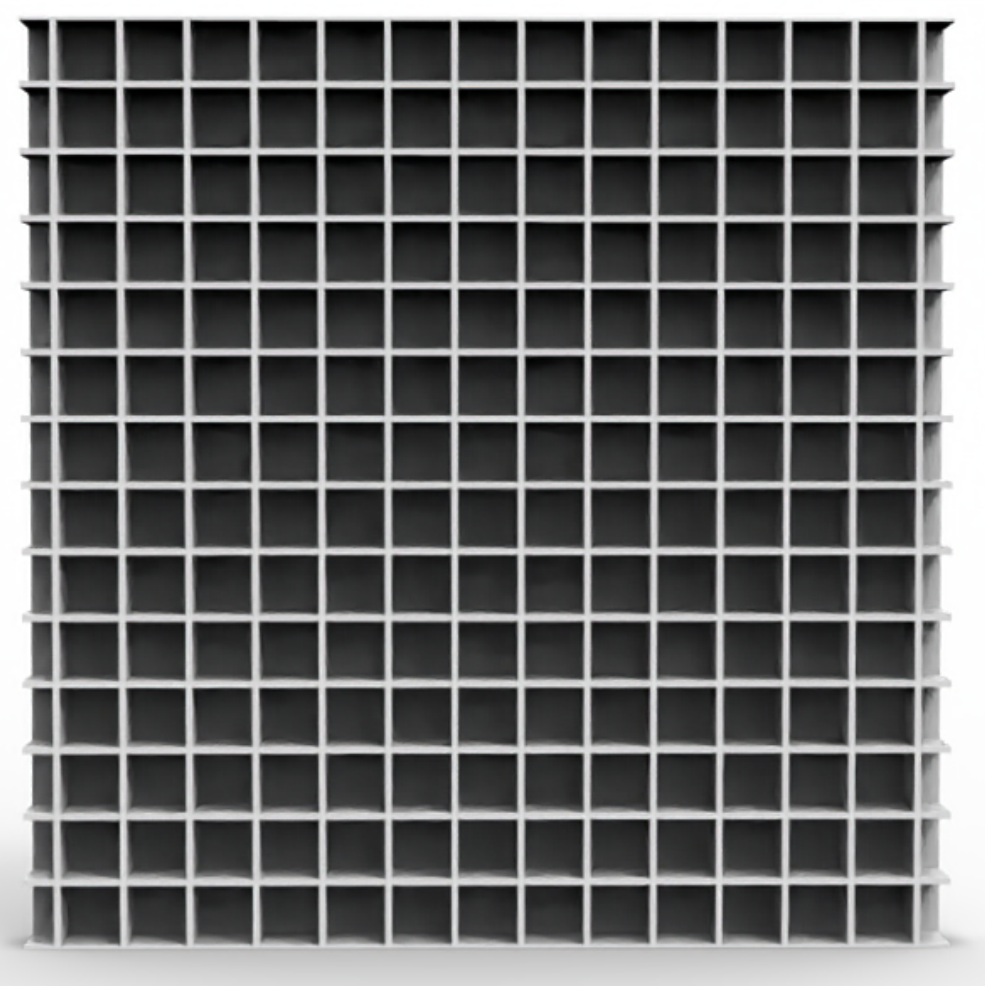

(a)

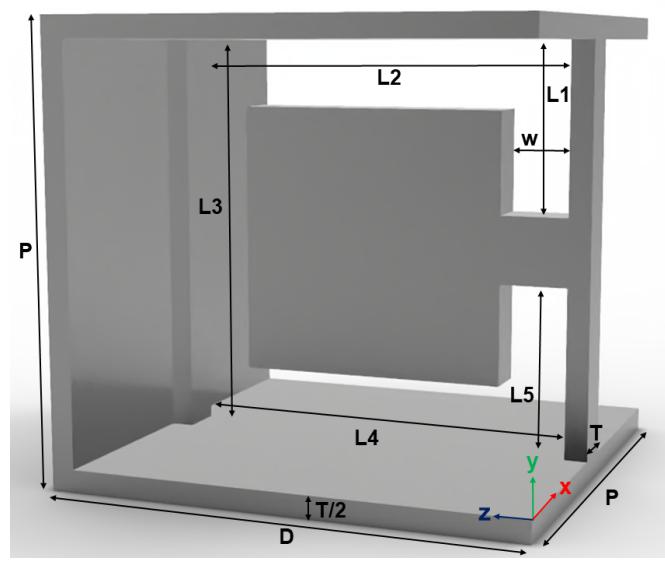

(b)

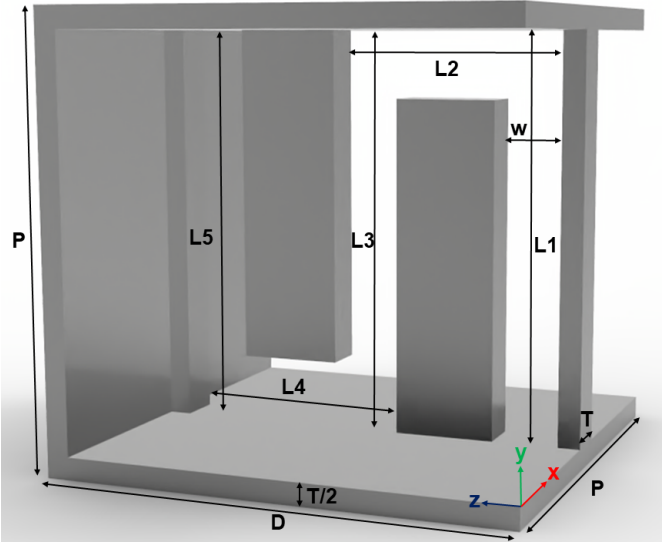

(c)

Figure 1. (a): Array $14 \times 14$ square waveguides. (b): Unit-cell type with a C-shaped resonator on the wall and dimensions. (c): Unit-cell type with an S-shaped resonator on the wall and dimensions. The length of the slot perforation is calculated as $L=L_{1}+L_{2}+L_{3}+L_{4}+L_{5}$.

\section{Performance of the Unit Cell. Choice of the Resonant Element}

The phase of the reflection coefficient associated with a particular cell is manipulated in terms of the resonant frequency of the resonator. In the RA-design context, the role of 
each cell is to provide the correct phase shift to redirect the incident beam to a desired (different) direction.

In order to study and analyze the phase behavior of each cell as a function of frequency, we must carry out a detailed study of the configuration of the resonator employed. The process involves modifying the geometry of the resonator so that it becomes smaller but while maintaining the same width value $w$ between its edges. To do this, we first define the effective length $L=L_{1}+L_{2}+L_{3}+L_{4}+L_{5}$ of the resonant element. In each iteration, we reduce this parameter so that, consequently, a phase curve is obtained, which shifts in frequency as we shorten the resonator.

The study of the phase is realized by computing the $S_{11}$-parameter of the cell when it is illuminated by a V-polarized plane wave. This study is focused on the Ku-band, specifically at frequencies close to $12.5 \mathrm{GHz}$. Though no propagating modes are excited inside the cells, the dominant (evanescent) mode is the $\mathrm{TE}_{10}$, whose electric field is oriented on the $\hat{y}$-axis and is responsible for excitation of the resonances. By modifying the dimensions of the resonator, the phase of the reflection coefficient can be controlled. A specific resonator geometry is characterized by a specific phase curve.

Figure 2a illustrates the phase variation as a function of frequency for C-and S-shaped resonators. The curves were obtained using the electromagnetic solver CST. Different curves, each associated with a particular geometry of the resonators, are appreciated. The first graphical difference that we observe corresponds to the slope of the curves of both resonators, being much greater for the S-shaped resonator, which implies a greater phase change in a smaller range of frequencies.

Additionally, taking the central frequency of the configuration as a reference $(12.5 \mathrm{GHz})$, small variations can be appreciated on the C-shaped resonator geometry, corresponding to small phase shifts at the reference frequency. S-shaped resonators are more sensitive, providing a stronger shifting even for small geometry variations. Therefore, considering the reference frequency at $12.5 \mathrm{GHz}$, greater phase variations are given with S-shaped slots, while smaller variations are associated with the $\mathrm{C}$-shaped ones, corresponding to smaller phase shifting.

The dependence between the geometry variation and the phase shift is clearer plotted in Figure $2 \mathrm{~b}$. The geometry variation is denoted by $\Delta L$, whereas the phase shift is identified as $\Delta$ Phase. The method to obtain these curves is straightforward due to Figure 2a. This is done by separately taking all the cut-off points of the phase curves, both of the Cand S-shaped resonators, with a vertical line marking the frequency of $12.5 \mathrm{GHz}$, which corresponds to the center frequency of the reflector. A soft interpolation was applied to the curves in order to achieve greater smoothing.

This graph gives us useful information about the phase variation obtained when we modify the effective length $L$. Analyzing, in detail, the behavior of these curves, we observe a very similar trend although with a different slope. For the final design of our reflective structure, we consider two different areas: the blue curve (corresponding to the C-shaped resonator) for the central cells and the orange curve (corresponding to the $\mathrm{S}$-shaped resonator) for the outermost cells.

Since the blue curve ( $C$ shape) has a lower slope, greater phase jumps are obtained as we reduce the geometry of the resonator. This is not the case of the S-shaped resonator because a very small change in $L$ (specially from $\Delta L=2.5 \mathrm{~mm}$ to $\Delta L=1.35 \mathrm{~mm}$ ) causes a large phase jump. It can also be inferred that both kind of resonators are necessary in order to cover an almost $360^{\circ}$-range. C-shaped resonators cover the central area, from $-200^{\circ}$ to $100^{\circ}$ approximately $\left(300^{\circ}\right.$ phase span), whereas the external area is covered by the S-shaped ones. This allows us to obtain a greater phase variation and to be able to reach the full range very close to $360^{\circ}$. 


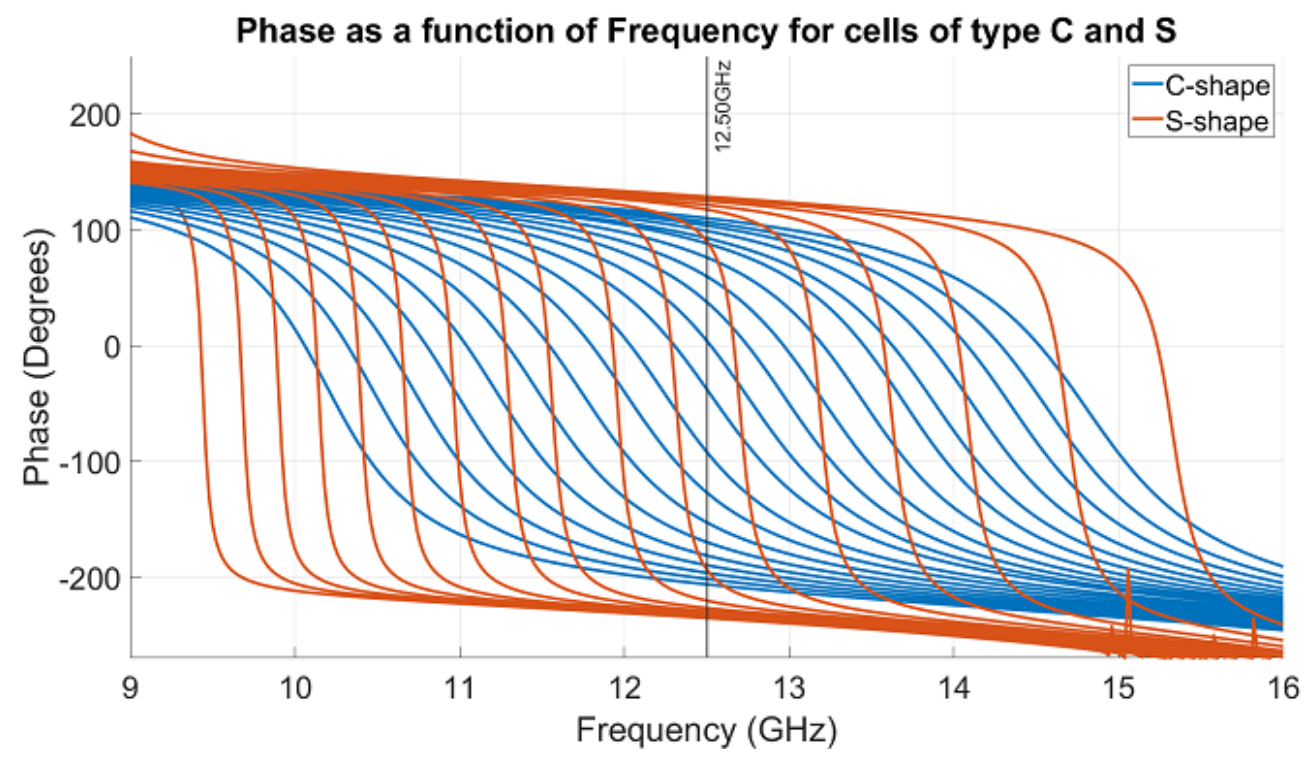

(a)

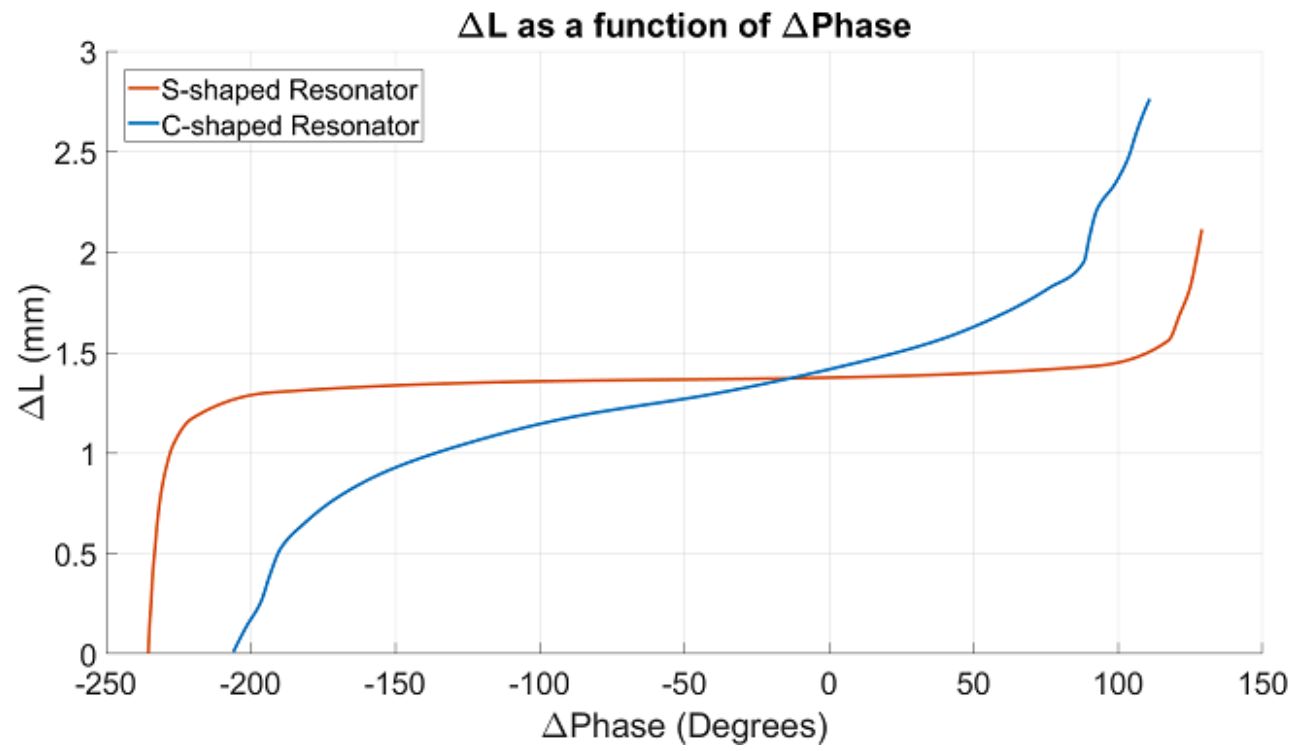

(b)

Figure 2. (a): Phase versus frequency for cells with $\mathrm{C}$-shaped and S-shaped resonators. (b): $\Delta \mathrm{L}$ versus $\Delta$ Phase for S-shaped and C-shaped resonators.

\section{Reflectarray Design: Numerical Validation}

The design plots in the previous section illustrate that the combination between Cand S-shaped perforations practically covers the $360^{\circ}$-phase range necessary to design a RA. At this point, and for fabrication purposes, we will restrict the phase gradient of our RA to a set of discrete values. This limitation is imposed by the tolerances of the 3D printer of our laboratory, which is a Form 3 from Formlabs. The information containing the features of the machine can be found in [37]. On the one hand, a gradient formed just by six possible states, whose corresponding phase values are defined by the following relation:

$$
\frac{n \pi}{3}, \quad n \in[0,5]
$$

On the other hand, a simple design based in just two phase-states: 0 and $\pi$, which will be encoded as 0 and 1, respectively. This is the so-called binary cell. 
The design and optimization of the RA is realized according to the scenario shown in Figure 3. A standard WR-75 horn antenna feeds the RA and is placed at $15 \mathrm{~cm}$ from it. This distance is optimal to reduce the spillover. The RA is a $14 \times 14$-cells panel with periodicity $P=10.53 \mathrm{~mm}$. Ray-tracing approximation is employed to estimate the phase arriving to each of the cells.

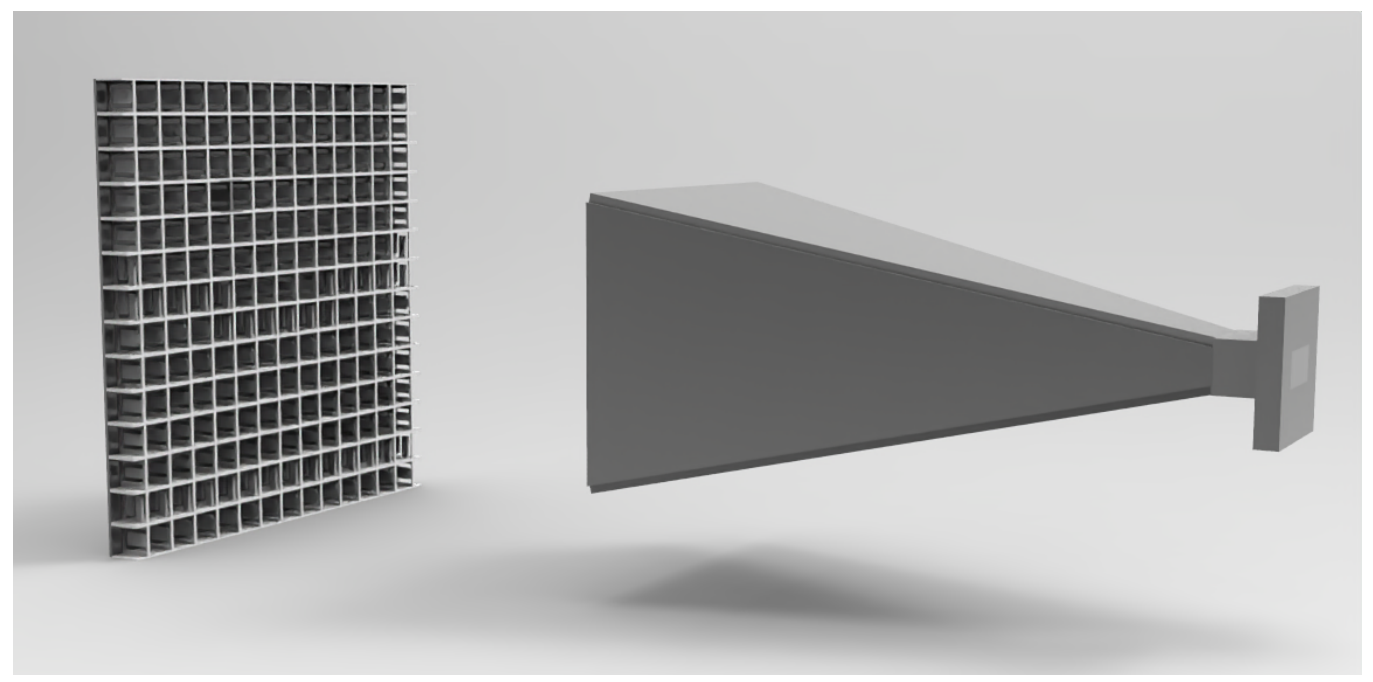

Figure 3. Scenario simulated in CST. The horn feeds the RA from a distance of $15 \mathrm{~cm}$. Structure parameters of the cells in the RA: $P=10.53 \mathrm{~mm}, T=0.5 \mathrm{~mm}, D=10 \mathrm{~mm}$.

The total phase in each cell is equal to the phase of the incident field (due to the propagation from the feed) plus the phase-shift introduced by the own cell. In this sense, the phase shift required at each element to re-direct the incident field is given by the following expression [4]:

$$
\begin{gathered}
\varphi_{n m}=k_{0}\left(d_{n m}-\left[x_{n} \cos \left(\varphi_{b}\right)+y_{n} \sin \left(\varphi_{b}\right)\right] \cos \left(\theta_{\mathrm{b}}\right)\right) \\
n, m=1, \ldots, 14
\end{gathered}
$$

where $\left(x_{n}, y_{m}\right)$ are the coordinates of the position of the $n m$ th RA-cell, $k_{0}$ is the wavenumber in vacuum, and $d_{n m}$ is the distance from the phase center of the feed to the nmth RA-cell. The parameters $\theta_{b}$ and $\varphi_{b}$ denote the outgoing polar and azimuthal angles respectively, namely, the pointing direction of the steered beam.

For the sake of simplicity, and as proof of concept, two arbitrary different pointing angles were chosen: $\theta_{b}=20^{\circ}$ and $\theta_{b}=35^{\circ}$ with $\varphi_{b}=0^{\circ}$, being the latter a check of the performance Assuming first a 6-states phase distribution, the phase-shift value satisfying (2) is approximated to the nearest phase-value in (1). The $14 \times 14$-panels for $\theta_{b}=20^{\circ}, 35^{\circ}$ are plotted in Figures $4 \mathrm{a}$ and $5 \mathrm{a}$, respectively. The corresponding radiation diagrams associated with these panels are plotted in orange lines in Figures $4 c$ and $5 c$, respectively. The main lobes are exactly at the desired angles, with a gain of 22.5 and $22.6 \mathrm{~dB}$ and a side-lodes level (SLL) of 12.5 and $11.8 \mathrm{~dB}$, respectively.

The RA based on binary cells was optimized using a Genetic Algorithm since an ad-hoc truncation can result in large phase errors. Specifically, NSGA-II was employed to reach our goal. The Non-dominated Sorting Genetic Algorithm, NSGA-II [38], was used, which is a multi-objective algorithm that has been applied in some of our previous jobs [35]. The optimization process is initialized with a random set of solutions, each one with $14^{2}$ binary variables $(0 / 1)$. The selection operator utilized is Binary Tournament, while the recombination (also known as crossover) consists in the exchange of random subzones of the phase configuration.

In this way, we provide diversity among the solutions, which is key for the algorithm to not stall. Finally, the mutation operator flips the value of a single bit, from 0 to 1 or vice 
versa. Previously, we attempted to flip a group of bits, but this prevented the algorithm from converging completely. The crucial component of a GA is the fitness function to optimize. Although this algorithm is multi-objective, in this functionality that we call beam steering, only one objective can be established. Thus, we will maximize the gain of the structure at the desired pointing direction.

We can observe, in Figures $4 \mathrm{~b}$ and $5 \mathrm{~b}$, the $14 \times 14$ phase-panel obtained for this case. As expected, the RA gained in simplicity. It is worth remarking that the phase evolution follows a similar tendency in both cases, simulating a sort of partial rings. The phase diversity is, as expected, richer in the six-state case. The simplicity of the one-bit RA is manifested in the results plotted in a blue curve in Figures $4 \mathrm{c}$ and $5 \mathrm{c}$. However, the gain decreases down to 20.8 and $20.7 \mathrm{~dB}$, whereas the SLL increases up to 15.5 and $15.4 \mathrm{~dB}$, respectively.

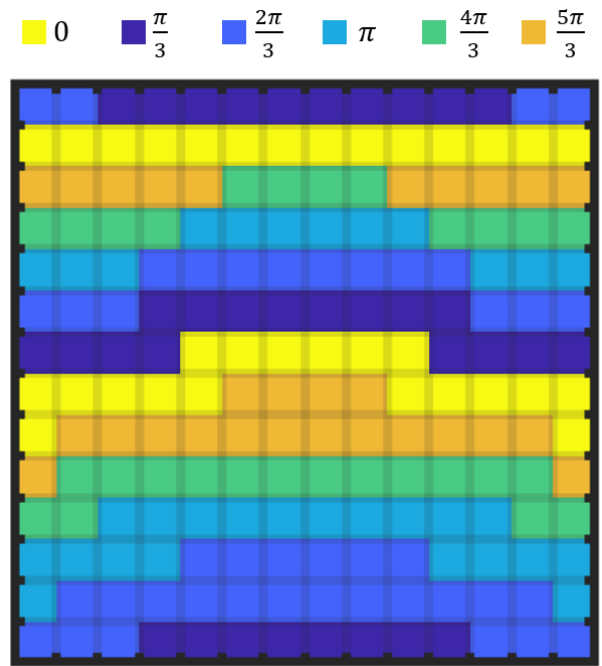

(a)

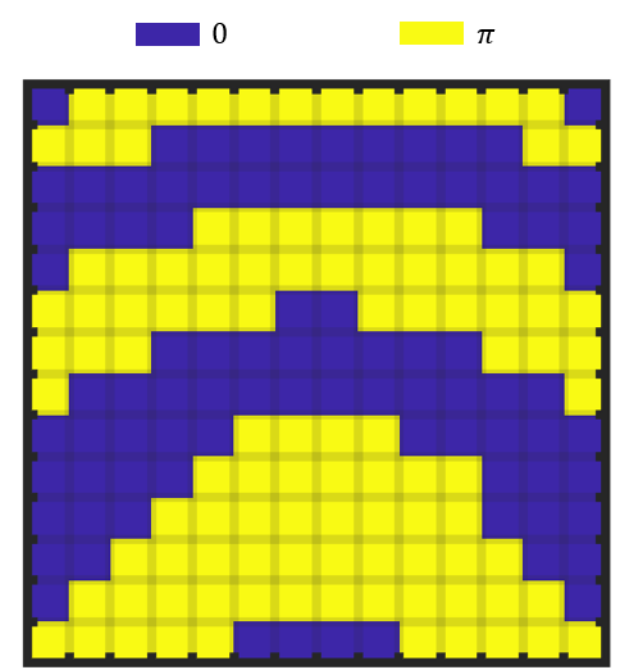

(b)

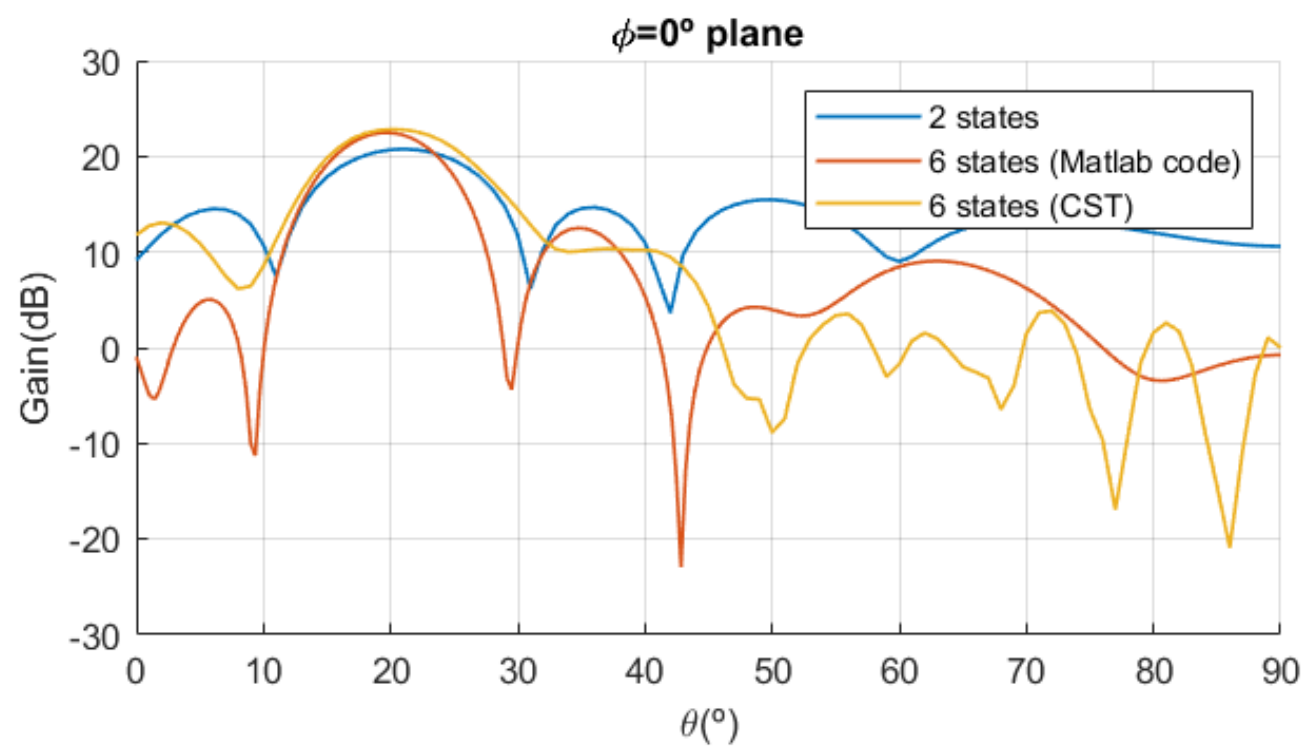

(c)

Figure 4. (a): Phase arrangement considering six states. (b): Phase arrangement considering two states. (c): Radiation pattern, beam direction: $(\varphi, \theta)=(0,20)^{\circ}$. 


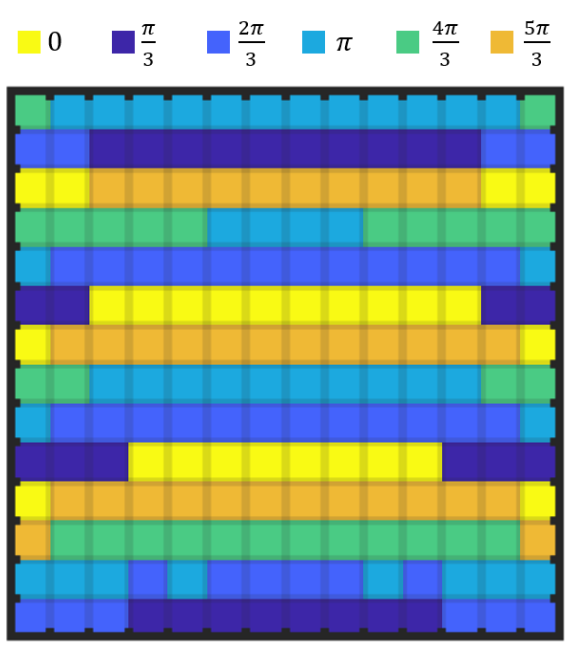

(a)

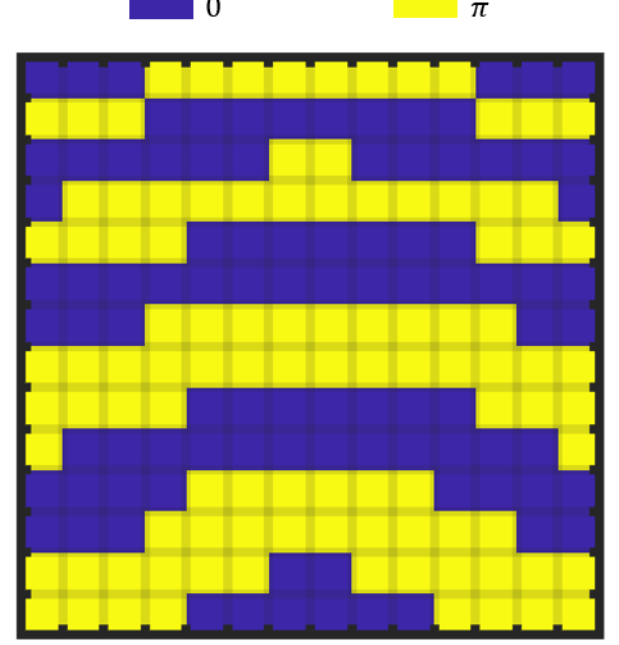

(b)

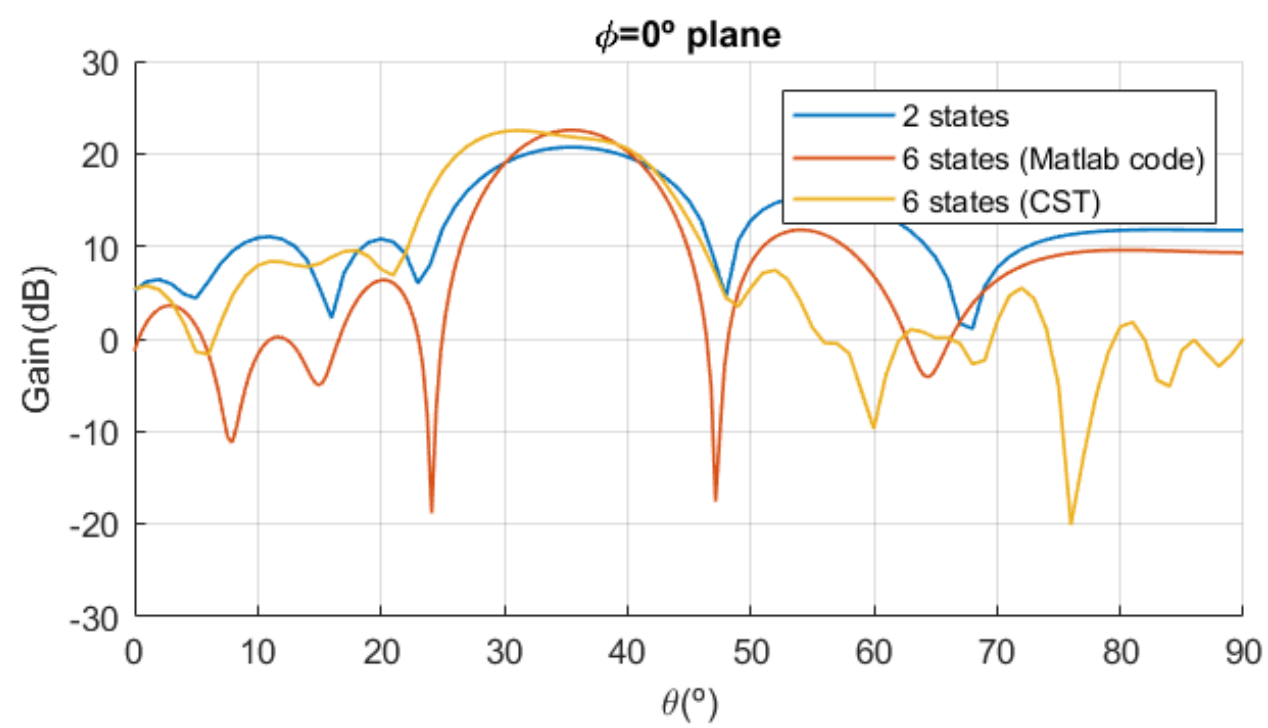

(c)

Figure 5. (a): Phase arrangement considering six states. (b): Phase arrangement considering two states. (c): Radiation pattern, beam direction: $(\varphi, \theta)=(0,35)^{\circ}$.

Finally, the scenario in Figure 3 was simulated by the commercial software CST. The RA choice is the one with six phase-states. The phase distribution in Figures $4 \mathrm{a}$ and $5 \mathrm{~b}$ are implemented in the RA with the help of the design plots in Figure 2a,b. The results of the simulation are also plotted in Figures $4 c$ and $5 c$ (yellow curves). Minor differences are appreciated due to the avoidance of possible mutual coupling among cells at the designing process.

The MATLAB code follows an equation, and that is why it appears as a smooth curve, while the simulation in CST, which is a full-wave electromagnetic simulator, takes into account possible mutual coupling among cells that are not considered the designing process. The SLL is otherwise still acceptable, with differences of more than $10 \mathrm{~dB}$ with respect the main lobe maximum. We cannot say which configuration is better, due to this trade-off between the gain obtained, which is better with six states, and the time spent to find the best configuration, which is less with binary states. 


\section{Manufacturing and Experimental Validation}

Once the design is optimized and completely adjusted to fulfill the requirements, the structure is manufactured. A $14 \times 14$ array prototype was fabricated. As a proof of concept, we decided to focus on the six-state RA operating for $\theta=20^{\circ}$. The manufacturing process was carried out due to the manufacturing facilities of the SWAT group of the University of Granada. The process implies accurate 3D stereolithography, consisting on the construction of the structure layer by layer by a photochemical process. The material is a resin characterized by $\varepsilon_{\mathrm{r}}=2.55-\mathrm{j} 0.0612$.

Since the dimensions of the entire RA are larger than recommended by the 3D printer, it was split and printed in nine individual pieces. Each of these individual pieces consisted of arrangements of $3 \times 3$ or $4 \times 4$ cells. Additional supports were needed in order to guarantee the self-robustness of the pieces during the fabrication process. Figure 6a shows the nine pieces including the supports, which are essentially very narrow dielectric bars. When the piece is completed, it is centrifuged and sunk in isopropyl alcohol in order to avoid resin remnants. It is also cured with ultraviolet light to completely fix the resin.

Then, the supports are carefully removed using special scissors. After that, all the pieces are glued together, giving rise to the entire RA. Finally, the metallization is realized by using a commercial conductive ink. In particular, this commercial ink is super-shield nickel coating aerosol (841AR from MG Chemicals), which was successfully employed in [39]. As previously mentioned, metallization (in this case via ink) is necessary since the RA is originally dielectric. Figure $6 \mathrm{~b}$ shows a detailed view of the final prototype. The presence of the ink can be inferred from the darker color of the structure.

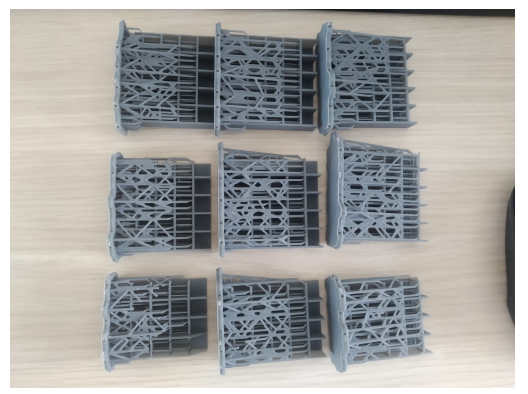

(a)

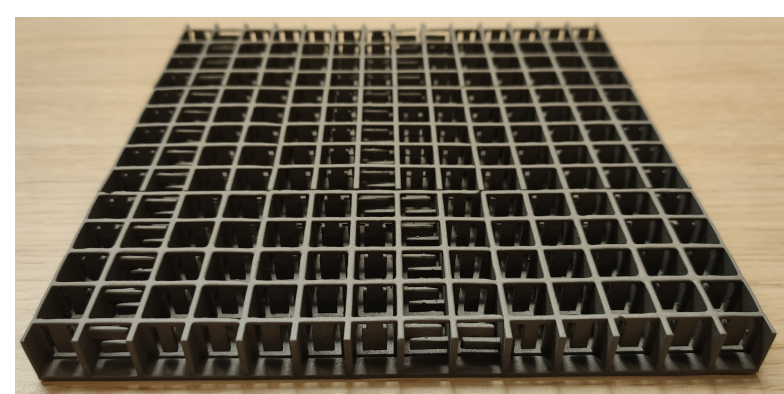

(b)

Figure 6. (a): Photo of the individual pieces including the supports. (b): Photo of the final RA prototype. The S- and C-shaped resonators can easily be visualized.

The structure was mounted in its setup with the feeder located at the defined distance of $15 \mathrm{~cm}$ and measured at the mmWave antenna measuring facility of the SWAT Group at the University of Granada. The RA setup can be seen in Figure 7a,b, while the measuring setup in the measuring system is depicted in Figure 7c. The prototype is finally characterized in terms of its radiation pattern in the vertical plane. The pattern measurement is shown in Figure 8, having the center working frequency at $12.7 \mathrm{GHz}$. This yields a frequency shift of $0.2 \mathrm{GHz}$ with respect to the theoretical results.

The position of the main lobe is approximately situated at the expected angle. There exist a small angular shift towards higher angles. Additionally, the secondary-lobes level is larger than expected from the simulations. It oscillates between -4 and $-10 \mathrm{~dB}$ in the region close to the main lobe position. This disagreement is important since it considerably reduces the efficiency of the RA. The source of inaccuracy is clearly the fabrication process. The structure is actually challenging from the printing process point of view. As we explained above, it was split into nine sections.

When the pieces are put together, small misalignments appear among all of them. Furthermore, the metallization via ink must be carefully taken into account. It is very important to metallize all of the structure. In the resonator regions, the conductive ink may not reach all the corners, reducing the efficiency of the resonator (longer or shorter than 
expected). Finally, the process of removing the additional supports is very cumbersome. This task is done manually.

Some portions of the supports may rest in the structure, affecting the phase shifting and the final far-field pattern. This RA is the first structure manufactured in our group. Currently, we are refining the technique and we expect to have better prototypes in the near future. We are also exploring how to metallize with copper. The results reported in this paper encourage us to continue.

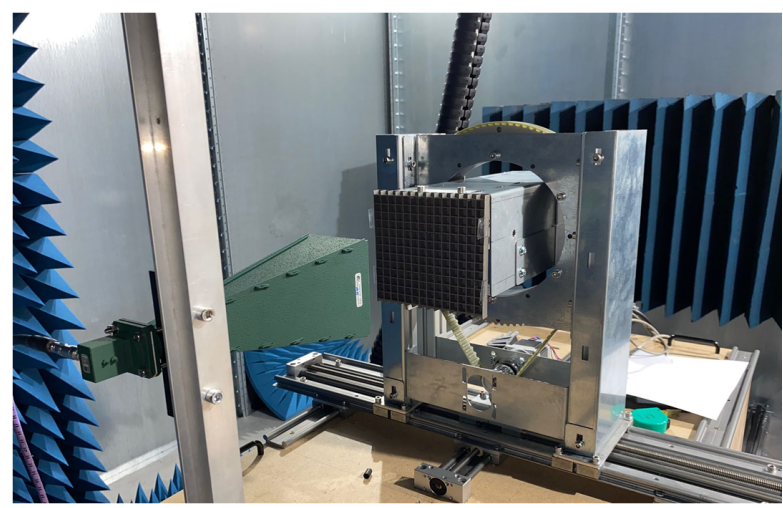

(a)

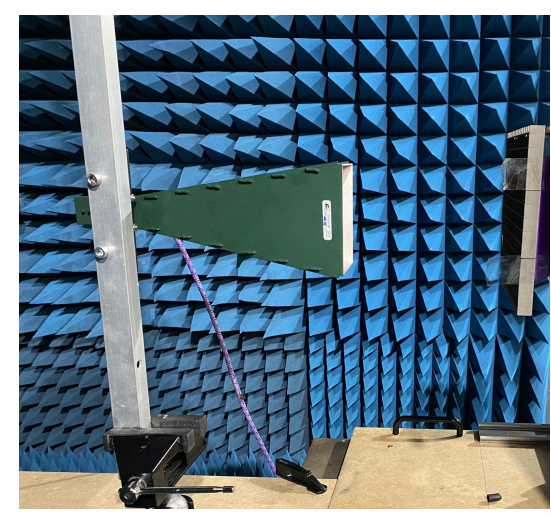

(b)

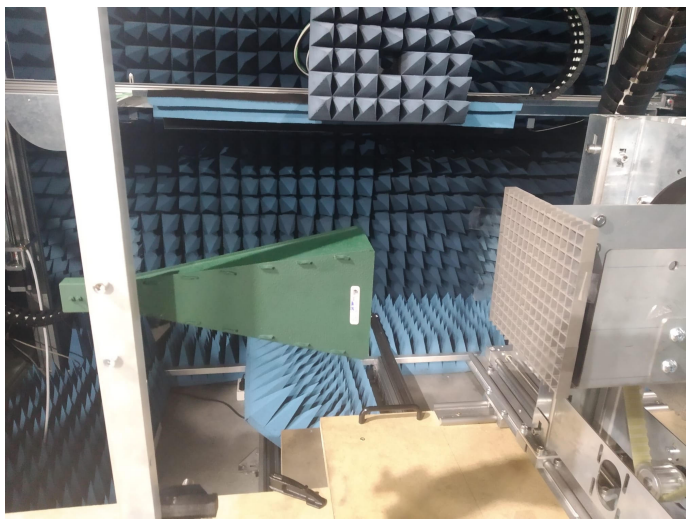

(c)

Figure 7. RA Antenna prototype mounted in its final configuration (a,b), and (c) measuring setup at the antenna measuring facility of the SWAT Group at the University of Granada.

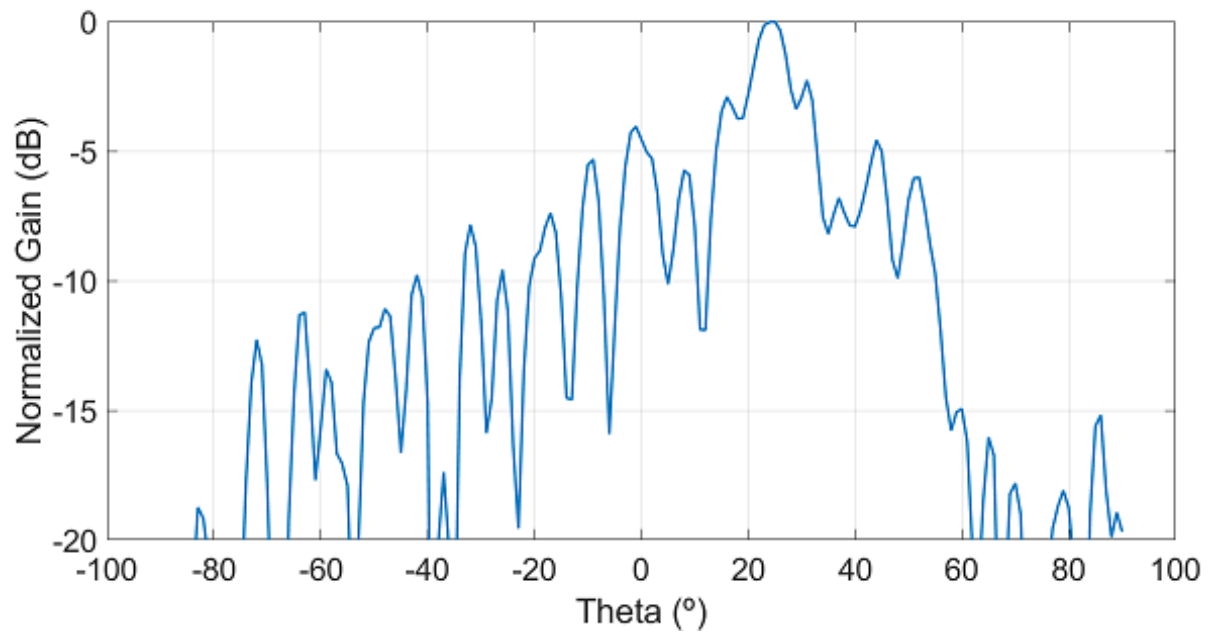

Figure 8. Radiation pattern in the vertical plane. Best result obtained at $12.7 \mathrm{GHz}$. 


\section{Conclusions}

This work presents the design and manufacture of a reflectarray (RA) antenna for the Ku-band, based on a fully-metallic 3D architecture. The structure used for the array structure is a FSS based on a fully-metallic design, which consists of a two-dimensional arrangement of classical square waveguides, with each of these waveguides being the unit cell of the whole structure. The cell is shorted by placing a ground plane at the bottom, in order to force a full-reflection response. Each cell has the ability of configuring the phase of its own reflected field by means of resonators perforated on the walls of the cell waveguide section. These perforations actually play the role of slots resonators, which drastically modify the electromagnetic response of the cell at frequencies close to their own resonance frequency.

In this manuscript, two main resonator-shapes are proposed (C-shaped and S-shaped slot resonators). The inclusion of resonant elements along the propagation direction permits the separate control of the vertical and horizontal field-polarizations. The designed structure proposed in this paper as a proof of concept is focused on the control of the V-pol incidence. This design explores the potential of phase value truncation (six states and two states) and demonstrates that proper pattern results can be obtained with this phase truncation. There is good agreement between the design outcomes and the measurement results of the design.

Author Contributions: Although all the authors has contributed to each section, J.V. has been fully involved with Sections 3 and 4; I.P.-S. with Section 4; and C.M. with Section 2. All authors have read and agreed to the published version of the manuscript.

Funding: This research received no external funding.

Acknowledgments: This work was supported in part by the Spanish Government under Project PID2020-112545RB-C54, Project RTI2018-102002-A-I00 and Project TIN2016-75097-P, in part by “Junta de Andalucía" under Project B-TIC-402-UGR18, A-TIC-608-UGR20 and Project P18.RT.4830, and in part by the Project associated with the Leonardo Scholarship 2021 for researchers and cultural creators from the BBVA foundation. The authors would like to thank the constructive comments and help of Pablo Padilla.

Conflicts of Interest: The authors declare no conflict of interest.

\section{References}

1. Gupta, A.; Jha, R.K. A Survey of 5G Network: Architecture and Emerging Technologies. IEEE Access 2015, 3, 1206-1232. [CrossRef]

2. Agiwal, M.; Kwon, H.; Park, S.; Jin, H. A Survey on 4G-5G Dual Connectivity: Road to 5G Implementation. IEEE Access 2021, 9, 16193-16210. [CrossRef]

3. Dahri, M.H.; Jamaluddin, M.H.; Abbasi, M.I.; Kamarudin, M.R. A Review of Wideband Reflectarray Antennas for 5G Communication Systems. IEEE Access 2017, 5, 17803-17815. [CrossRef]

4. Huang, J.; Encinar, J.A. Introduction to Reflectarray Antenna; Wiley-IEEE Press: New York, NY, USA, 2008.

5. Katagi, T.; Chiba, I. Review on recent phased array antenna technologies in Japan. In Proceedings of the IEEE Antennas and Propagation Society International Symposium. Transmitting Waves of Progress to the Next Millennium. 2000 Digest. Held in Conjunction with: USNC/URSI National Radio Science Meeting (Cat. No.00CH37118), Salt Lake City, UT, USA, 16-21 July 2000; Volume 2, pp. 570-573.

6. Samejima, S. Phased array antenna systems for commercial applications in Japan. In Proceedings of the International Symposium on Phased Array Systems and Technology, Boston, MA, USA, 15-18 October 1996.

7. Rotman, R.; Tur, M. Antenna and beamformer requirements for wideband phased array systems: A review. In Proceedings of the 2009 IEEE International Conference on Microwaves, Communications, Antennas and Electronics Systems, Tel Aviv, Israel, 9-11 November 2009.

8. Chen, P.; Hong, W. Research advances in phased array antennas for satellite communications. In Proceedings of the 12th European Conference on Antennas and Propagation (EuCAP 2018), London, UK, 9-13 April 2018.

9. Mailloux, R. Phased Array Antenna Handbook, 3rd ed.; Artech: Norwood, MA, USA, 2017.

10. Rauf, A.A.; Tahir, J.; Raza, A.; Ali, A.; Umrani, I.H. 16 ways X-band wilkinson power divider for phased array transmitter. In Proceedings of the 2018 15th International Bhurban Conference on Applied Sciences and Technology (IBCAST), Islamabad, Pakistan, 9-13 January 2018; pp. 835-840. 
11. Zhang, Q.; Yuan, C.; Liu, L. Studies on mechanical tunable waveguide phase shifters for phased-array antenna applications. In Proceedings of the 2016 IEEE International Symposium on Phased Array Systems and Technology (PAST), Waltham, MA, USA, 18-21 October 2016.

12. Pozar, D.M. The active element pattern. IEEE Trans. Antennas Propag. 1994, 42, 1176-1178. [CrossRef]

13. Nayeri, P.; Yang, J.; Elsherbeni, A.Z. Reflectarray Antennas: Theory, Designs, and Applications; Wiley-IEEE Press: New York, NY, USA, 2018.

14. Berry, D.; Malech, R.; Kennedy, W. The reflectarray antenna. IEEE Trans. Antennas Propag. 1963, 11, 645-651. [CrossRef]

15. Encinar, J.A. Design of two-layer printed reflectarrays using patches of variable size. IEEE Trans. Antennas Propag. 2001, 49, 1403-1410. [CrossRef]

16. Arrebola, M.; Encinar, J.A.; Barba, M. Multifed Printed Reflectarray With Three Simultaneous Shaped Beams for LMDS Central Station Antenna. IEEE Trans. Antennas Propag. 2008, 56, 1518-1527. [CrossRef]

17. Robustillo, P.; Zapata, J.; Encinar, J.A.; Arrebola, M. Design of a Contoured-Beam Reflectarray for a EuTELSAT European Coverage Using a Stacked-Patch Element Characterized by an Artificial Neural Network. IEEE Antennas Wirel. Propag. Lett. 2012, 11, 977-980. [CrossRef]

18. Florencio, R.; Encinar, J.A.; Boix, R.R.; Losada, V.; Toso, G. Reflectarray Antennas for Dual Polarization and Broadband Telecom Satellite Applications. IEEE Trans. Antennas Propag. 2015, 63, 1234-1246. [CrossRef]

19. Li, B.; Mei, C.Y.; Zhou, Y.; Lv, X. A 3-D-Printed Wideband Circularly Polarized Dielectric Reflectarray of Cross-Shaped Element. IEEE Antennas Wirel. Propag. Lett. 2020, 19, 1734-1738. [CrossRef]

20. Mei, P.; Zhang, S.; Pedersen, G.F. A Low-Cost, High-Efficiency and Full-Metal Reflectarray Antenna With Mechanically 2-D Beam-Steerable Capabilities for 5G Applications. IEEE Trans. Antennas Propag. 2020, 68, 6997-7006. [CrossRef]

21. Henderson, K.Q.; Ghalichechian, N. Circular-Polarized Metal-Only Reflectarray With Multi-Slot Elements. IEEE Trans. Antennas Propag. 2020, 68, 6695-6703. [CrossRef]

22. Deng, R.; Yang, F.; Xu, S.; Li, M. A Low-Cost Metal-Only Reflectarray Using Modified Slot-Type Phoenix Element With $360^{\circ}$ Phase Coverage. IEEE Trans. Antennas Propag. 2016, 64, 1556-1560. [CrossRef]

23. Yi, M.; Bae, Y.; Yoo, S.; So, J. Digitized Reconfigurable Metal Reflectarray Surfaces for Millimeter-Wave Beam-Engineering. Appl. Sci. 2021, 11, 5811. [CrossRef]

24. Zhu, D.Z.; Gregory, M.D.; Werner, P.L.; Werner, D.H. Fabrication and Characterization of Multiband Polarization Independent 3-D-Printed Frequency Selective Structures With UltraWide Fields of View. IEEE Trans. Antennas Propag. 2018, 66, 6096-6105. [CrossRef]

25. Rashid, A.K.; Shen, Z. Three-dimensional frequency selective surfaces. In Proceedings of the 2010 International Conference on Communications, Circuits and Systems (ICCCAS), Chengdu, China, 28-30 July 2010; pp. 688-691. [CrossRef]

26. Pelletti, C.; Bianconi, G.; Mittra, R.; Shen, Z. Frequency selective surface with wideband quasi-elliptical bandpass response. Electron. Lett. 2013, 49, 1052-1053. [CrossRef]

27. Omar, A.A.; Shen, Z. Multiband High-Order Bandstop 3-D Frequency-Selective Structures. IEEE Trans. Antennas Propag. 2016, 64, 2217-2226. [CrossRef]

28. Molero, C.; Debogovic, T.; García-Vigueras, M. Design of Full-Metal Polarizing Screen Based on Circuit Modeling. In Proceedings of the 2018 IEEE/MTT-S International Microwave Symposium-IMS, Philadelphia, PA, USA, 10-15 June 2018; pp. 23-26.

29. Jimenez, C.M.; Menargues, E.; García-Vigueras, M. All-Metal 3-D Frequency-Selective Surface With Versatile Dual-Band Polarization Conversion. IEEE Trans. Antennas Propag. 2020, 68, 5431-5441. [CrossRef]

30. Rajagopalan, H.; Rahmat-Samii, Y. Dielectric and Conductor Loss Quantification for Microstrip Reflectarray: Simulations and Measurements. IEEE Trans. Antennas Propag. 2008, 56, 1192-1196. [CrossRef]

31. Wilke, R. Radiation Effects in PTFE based RF Substrates to be Used as Antennas in Geostationary Orbits. In Proceedings of the 2014 DLR Bauteile Konferenz, Freiburg, Germany, 9-10 April 2014.

32. Wilke, R.; Schraml, K.; Heberling, D. Space radiation hardness of PTFE based RF substrates for GEO satellite application. In Proceedings of the 2015 9th European Conference on Antennas and Propagation (EuCAP), Lisbon, Portugal, 13-17 April 2015; pp. $1-5$.

33. Chahat, N.; Cook, B.; Lim, H.; Estabrook, P. All-Metal Dual-Frequency RHCP High-Gain Antenna for a Potential Europa Lander. IEEE Trans. Antennas Propag. 2018, 66, 6791-6798. [CrossRef]

34. Dai, J.Y.; Tang, W.; Chen, M.Z.; Chan, C.H.; Cheng, Q.; Jin, S.; Cui, T.J. Wireless Communication Based on Information Metasurfaces. IEEE Trans. Microw. Theory Tech. 2021, 69, 1493-1510. [CrossRef]

35. Molero, C.; Palomares, A.; Álex, A.; Parellada, I.; Gámiz, F.; Padilla, P.; Valenzuela, J.F. Metamaterial-Based Reconfigurable Intelligent Surface: 3D Meta-Atoms Controlled by Graphene Structures. IEEE Commun. Mag. 2021, 59, 42-48. [CrossRef]

36. Tang, W.; Zhu, J.; Wang, C.; Ge, J.; Yu, Z.; Zhuang, W. Waveguide 3-D FSSs by 3-D printing technique. In Proceedings of the 2016 International Conference on Electromagnetics in Advanced Applications (ICEAA), Cairns, QLD, Australia, 19-23 September 2016; pp. 675-678. [CrossRef]

37. Available online: https://formlabs.com/es/3d-printers/form-3/ (accessed on 22 October 2021). 
38. Deb, K.; Pratap, A.; Agarwal, S.; Meyarivan, T. A Fast and Elitist Multiobjective Genetic Algorithm: NSGA-II. IEEE Trans. Evol. Comput. 2002, 6, 182-197. [CrossRef]

39. Rebollo, A.; Vaquero, Á.F.; Arrebola, M.; Pino, M.R. 3D-Printed Dual-Reflector Antenna With Self-Supported Dielectric Subreflector. IEEE Access 2020, 8, 209091-209100. [CrossRef] 